\title{
Evaluación del desempeño de la red LTE para la entrega del servicio de IPTV'
}

\section{Performance Evaluation of LTE Network for IPTV Service Delivery ${ }^{2}$}

\author{
Diego Fernando Rueda Pepinosa ${ }^{3}$ \\ Zoila Inés Ramos Rodríguez ${ }^{4}$
}

doi:10.11144/Javeriana.IYU18-2.edrl

Cómo citar este artículo:

RUEDA PEPINOSA, D. F. y RAMOS RODRÍGUEZ, Z. I. Evaluación del desempeño de la red LTE para la entrega del servicio de IPTV. Ingeniería y Universidad. 2014, vol. 18, no. 2, pp. 393-417. http://dx.doi.org/10.11144/Javeriana.IYU18-2.edrl

\footnotetext{
' Fecha de recepción: 11 de agosto de 2013. Fecha de aceptación: 31 de julio de 2014. Este artículo se deriva de un proyecto de investigación denominado Marco de referencia técnico para el despliegue del servicio de IPTV sobre redes móviles LTE (Long Term Evolution) con calidad de servicio (QoS). Este proyecto fue desarrollado por el Grupo de Investigación en Teleinformática de la Universidad Nacional de Colombia (GITUN), Bogotá, Colombia.

${ }^{2}$ Reception date: August $11^{\text {th }}, 2013$. Acceptance date: July 31 ${ }^{\text {st }}, 2014$. This article is the result of the research project Technical Framework for Deployment of IPTV Service on LTELTE (Long Term Evolution) Mobile Networks with Quality of Service (QOS), developed by the research group by Grupo de Investigación en Teleinformática de la Universidad Nacional de Colombia (GITUN), Bogotá, Colombia.

${ }^{3}$ Magíster en Ingeniería de Telecomunicaciones, Universidad Nacional de Colombia. Investigador del Grupo en Teleinformática de la Universidad Nacional de Colombia (GITUN), Bogotá, Colombia.Correo electrónico: dfruedap@unal.edu.co ${ }^{4} \mathrm{PhD}$ (c) en Ingeniería de Telecomunicaciones, Universidad de Telecomunicaciones e Informática de Moscú, Rusia. Profesora asociada. Líder del Grupo de Investigación en Teleinformática de la Universidad Nacional de Colombia (GITUN).

Correo electrónico: ziramosd@unal.edu.co
} 


\section{Resumen}

En este artículo se presentan los resultados obtenidos de la simulación de tráfico del servicio de IPTV móvil en un modelo de red LTE. Tal modelo se generó a partir de una arquitectura funcional para la entrega del servicio de IPTV sobre esta tecnología. Para la simulación se planteó un escenario donde los recursos de la red se comparten entre aplicaciones y servicios móviles (voz, video y datos), a cada uno de los cuales se les asignó la configuración de los parámetros de calidad de servicio necesarios para satisfacer sus requisitos de calidad de servicio. En dicho escenario se evaluó el comportamiento de los principales parámetros de desempeño de la red (retardo, razón de pérdida de paquetes y throughput) ante variaciones en la cantidad de terminales móviles conectados a la red, diferentes niveles de movilidad de los usuarios y configuración de algoritmos de planificación de tráfico bien conocidos de la red LTE. Como principal resultado se presenta el algoritmo que ofrece el mejor comportamiento de los parámetros de desempeño para tráfico de IPTV.

\section{Palabras clave}

calidad de servicio; IPTV móvil; red LTE

\section{Abstract}

This paper presents results about traffic simulation of mobile IPTV service in a LTE network model. The model is generated from a functional architecture for delivery of IPTV service over this technology. For the simulation is proposed a scenario where resources of network are shared between mobile services and applications: voice, video and data, each are assigned the necessary configuration of quality of service (QoS) parameters for satisfy their requirements of quality of service. In this scenario is evaluated the behavior of main performance parameters (delay, packet loss ratio, and throughput) against variations in the number of mobile terminals connect to the network, different levels of users mobility and configuration of well know scheduling algorithms of LTE network. As a main result the algorithm that gives the best behavior of the performance parameters for IPTV traffic is presented.

\section{Keywords}

quality of Service (QoS); LTE network; mobile IPTV 


\section{Introducción}

En el Internet Protocol Television (IPTV) la calidad de los contenidos entregados a los usuarios se ve afectada por diferentes parámetros de desempeño de la red que deben asegurarse en sus valores mínimos para garantizar la calidad percibida por los usuarios. El despliegue del servicio de IPTV sobre redes inalámbricas y móviles ha enfrentado serios desafíos, debido a las limitaciones de ancho de banda, a la baja fiabilidad y calidad de los enlaces de comunicaciones inalámbricos y a la movilidad de los terminales (Rueda y Ramos, 2012). Sin embargo, las últimas tecnologías inalámbricas de banda ancha se perfilan como redes idóneas para el despliegue de este servicio, toda vez que son arquitecturas completamente IP (all-IP) con capacidades de ofrecer calidad de servicio de extremo a extremo e incrementan la velocidad de acceso (Rueda y Ramos, 2013a).

Las redes Long Term Evolution (LTE) y LTE-Advanced (LTE-A) se constituyen en la evolución de las redes de tercera generación (3G) y se conciben como una infraestructura capaz de soportar servicios multimedia, porque ofrecen los recursos necesarios para garantizar la calidad en su entrega. En redes LTE, el modelo de calidad de servicio se basa en el uso de servicios portadores tipo Envolved Packet System (EPS), los cuales se clasifican como portadores por defecto, para transportar datos relacionados con señalización, control y de aplicaciones que no necesitan calidad de servicio, o como portadores dedicados, para transportar los flujos de tráfico asociados a servicios y aplicaciones con requisitos de calidad de servicio (Ekstrom, 2009). Los portadores por defecto no garantizan la tasa de bit, mientras que los portadores dedicados pueden o no garantizarla, dependiendo de los servicios y aplicaciones que transporten (Ekstrom, 2009).

En cuanto a la diferenciación del tráfico en las redes LTE, esta se realiza mediante la clasificación de servicios en clases, cada una de las cuales forma un flujo de paquetes al cual se le asignarán los recursos de la red según las exigencias del servicio usando portadores dedicados (Ekstrom, 2009). En este sentido, según 3GPP (2012a), desde la perspectiva del usuario, las clases de servicios son conversacional, interactiva, streaming y background. Las clases conversacional y 
streaming están destinadas para transportar tráfico de servicios de tiempo real, los cuales dependen directamente de la percepción humana y son muy sensibles al retardo (3GPP, 2012a). Entre tanto, las clases interactivas y background están pensadas para ser usadas por las aplicaciones de datos donde se debe garantizar una transferencia libre de errores sin tener en cuenta el retardo (3GPP, 2012a). Para identificar la clase de calidad de servicio en un servicio portador EPS se tienen los parámetros QoS Class Identifier (QCI) y Allocation and Retention Priority (ARP). Con este último se puede decidir si un servicio portador EPS puede establecerse en caso de congestión del canal de radio (Ekstrom, 2009).

Otro de los conceptos relacionados con la provisión de calidad de servicio en redes LTE tiene que ver con los algoritmos de planificación (scheduling) de paquetes tanto en el Downlink (DL) como en el Uplink (UL). Tales algoritmos permiten que los recursos de la red (portadores, tasas de bit y prioridades) sean asignados a cada uno de los flujos de tráfico teniendo en cuenta la calidad de servicio esperada, el comportamiento de las fuentes de datos, la carga de tráfico y la calidad del canal de radio. Por ello en diversos trabajos se han realizado estudios comparativos y propuesto algoritmos para la planificación de recursos en redes LTE con el objetivo de satisfacer los parámetros de desempeño de los diferentes servicios y aplicaciones que pueden circular por este tipo de redes (Rueda y Ramos, 2013a).

En este orden de ideas, en Ramli et al. (2009), los autores encontraron, mediante la evaluación del desempeño de los planificadores de paquetes bien conocidos para servicios de video streaming, que el algoritmo Maximum-Largest Weighted Delay First (M-LWDF) proporciona una baja razón de pérdida de paquetes y el mayor throughput al DL de la red LTE, que soporta un mayor número de usuarios que garantiza la equidad (fairness) en un grado satisfactorio. A pesar de que el algoritmo Exponential Proportional Fair (EXP/PF) tiene un buen throughput, la equidad es muy pobre si se compara con la obtenida con M-LWDF cuando el número de usuarios crece (Ramli et al., 2009).

Por otro lado, en Balint, Budura y Marza (2010) se analizaron otros planificadores de tráfico como Round Robin (RR) y Best Channel Quality Indicator (CQI) para una carga de tráfico mixto (voz y video streaming) en la red LTE, y se encontró que el planificador Best CQI ofrece una mejor experiencia a los usuarios que el algoritmo RR. Best CQI asigna un número apropiado de portadores de radio (Radio Bearer [RB]) para los usuarios de voz maximizando el número restante de recursos a los usuarios de video; el algoritmo Best CQI concede el número apropiado de RB para los usuarios de video que tiene un buen CQI, y aquellos 
que tienen un bajo CQI son rechazados por el planificador. En Basukala et al. (2010) se analiza el impacto del retardo del reporte no periódico del indicador de calidad de canal (CQI) sobre el rendimiento del algoritmo M-LWDF para atender a los usuarios de video streaming a diferentes velocidades. Los resultados de este trabajo recomiendan que para mantener la calidad de servicio a los usuarios que viajan a altas velocidades el reporte del CQI se debe hacer con mayor frecuencia que aquellos que se desplazan a menor velocidad.

En Piro et al. (2011a) se presenta una herramienta para la simulación de la red LTE en situaciones en las que se desean evaluar los parámetros de desempeño de la red. Para validar esta herramienta de simulación los autores plantean un escenario multicelda donde evaluaron el desempeño (retardo, razón de pérdida de paquetes y throughput) de las aplicaciones (voz, video y flujo de datos best effort [BE]) para cada uno de los planificadores de tráfico bien conocidos. Los resultados muestran que los algoritmos M-LWDF y EXP/PF mantienen una calidad de servicio adecuada para las aplicaciones en tiempo real como Voice over IP (VoIP) y video ante el crecimiento en el número de usuarios y velocidad de desplazamiento (3 y $120 \mathrm{~km} / \mathrm{h}$ ). Por su parte, en Piro et al. (2011b) se usa la herramienta propuesta en Piro et al. (2011a) para evaluar el desempeño de un planificador de tráfico de dos capas, con el cual se obtuvieron mejores resultados de desempeño de la red LTE para las aplicaciones en tiempo real que con los planificadores bien conocidos.

En el trabajo realizado por Cheng y Mohapatra (2012), a diferencia de las reglas de planificación convencionales que explotan las métricas de capa de red, el esquema propuesto por los autores se enfoca directamente en la optimización de la calidad de video en la capa de aplicación en el retardo de extremo a extremo requerido. La planificación de la calidad de video es tratada como un problema de optimización complejo, por lo cual su solución se basa en el enfoque metaheurístico del algoritmo genético. Como resultado, y al comparar con el algoritmo M-LWDF, se encuentra que con la solución del algoritmo genético la calidad de video se puede mejorar de manera significativa y satisfacer el retardo.

En otras investigaciones se ha estudiado la calidad de la experiencia en la entrega de video sobre redes LTE. Así, en Gao et al. (2013) se propone un algoritmo de planificación de paquetes para redes convergentes heterogéneas (LTE, WiFi, $2 \mathrm{G}$ y $3 \mathrm{G}$ ) con múltiples modos de comunicación que coexisten en un escenario de alta velocidad $(350 \mathrm{~km} / \mathrm{h})$. Los autores usan un mecanismo de mapeo de recursos virtual que considera diferentes niveles de prioridad, SINR, QCI y retardo en un subsistema de comunicaciones montado sobre un vehículo para garantizar 
la calidad de servicio a los flujos de voz, video y datos. Los resultados muestran que dicho algoritmo puede mejorar la experiencia del usuario y la eficiencia de transmisión, puesto que el throughput del sistema aumenta y la razón de pérdida de paquetes disminuye.

En Kumar y Oyman (2013) se estudia y analiza la calidad de la experiencia del usuario final para el servicio de video streaming, el cual está codificado en H.264/AVC y es entregado mediante enhanced Multicast/Broadcast Multimedia Service (eMBMS). Específicamente, la calidad de la experiencia se evalúa en términos del retardo de inicio de reproducción del video, el porcentaje de rebuffering y Peak Signal-To-Noise Ratio (PSNR).

En Shehada et al. (2013) se propone un esquema de calidad de la experiencia que estáticamente o dinámicamente reserva los recursos con el fin de mantener imperceptible la variación de la calidad durante el handover para la entrega de video sobre redes LTE. A través de simulaciones, se muestra que la reasignación de los recursos inalámbricos de manera cross-layer no es suficiente para mantener la calidad de video. Entonces, para disminuir la reserva de recursos innecesarios, los autores plantean hacerla de forma dinámica bajo el conocimiento previo del handover. Los resultados muestran que los esquemas propuestos conducen a mejoras significativas de calidad percibida por el usuario en el handover. Para un mayor detalle del estado de la cuestión de la implementación del servicio de IPTV sobre redes LTE con calidad de servicio se puede revisar el trabajo de Rueda y Ramos (2013a).

Si bien los trabajos encontrados en la literatura se encargan de evaluar la calidad de servicio o la calidad de la experiencia para la entrega de contenidos de video en diferentes entornos, esta investigación se diferencia de estos, pues aquí se presentan los resultados de la simulación de tráfico del servicio de IPTV móvil en un modelo de red LTE que está basado en la arquitectura funcional presentada en Rueda y Ramos (2012). En el escenario construido se evalúa el comportamiento de los principales parámetros de desempeño de la red (retardo, razón de pérdida de paquetes y throughput) ante variaciones en la cantidad de terminales móviles conectados a la red, diferentes niveles de movilidad de los usuarios y configuración de algoritmos de planificación de tráfico bien conocidos de la red. En este sentido, el presente trabajo está organizado de la siguiente manera: en la sección 1 se describe el escenario de simulación, el modelo de red LTE y los modelos de tráfico de las aplicaciones de voz, IPTV y datos; seguidamente en la sección 2 se presentan los resultados de la simulación, y en la última sección se presentan las conclusiones obtenidas con el desarrollo de esta investigación. 


\section{Descripción del experimento}

Luego de analizar en Rueda y Ramos (2013b) las características y alternativas de funcionamiento de la red LTE para la implementación del servicio de IPTV móvil con calidad de servicio, se eligió la herramienta LTE-Sim (Piro et al., 2011a) para la simulación del escenario propuesto para la evaluación de los parámetros de desempeño de la red (retardo, razón de pérdida de paquetes y throughput) frente a variaciones en la cantidad de terminales móviles conectados a la red para diferentes niveles de movilidad y configuración de calidad de servicio de la red LTE en un entorno multicelda.

La simulación del tráfico de IPTV móvil se planteó sobre un modelo de red LTE que se definió a partir de la arquitectura funcional propuesta en Rueda y Ramos (2012) y de los recursos ofrecidos por la herramienta LTE-Sim. De dicha arquitectura no se simuló el núcleo de IP Multimedia Subsystem (IMS), puesto que la simulación inicia con la transferencia de los flujos de datos de los servicios sin pasar por el proceso registro de usuarios y el proceso de descubrimiento y selección del servicio; la entidad Policy and Charging Rules Function (PCRF), ya que las reglas Policy and Charging Control (PCC) se configuran en los RB soportados por los envolved Node B (eNB); la pasarela Packet Data Network-Gateway (PDN-GW), debido a que corresponde a un equipo ajeno a la red LTE y no es del interés evaluar su desempeño, y los servidores de servicios y aplicaciones, porque el eNB se conecta directamente con el generador de tráfico de los servicios consumidos por los UE. En cuanto a los servicios de IPTV, se simula el servicio de IPTV de video bajo demanda $(\mathrm{VoD})$.

\subsection{Descripción del escenario de simulación}

La simulación del tráfico generado por un servicio de IPTV se realizó en un escenario multicelda/multiusuario (figura 1). En este se definió un entorno de red conformado por 9 celdas de $1 \mathrm{~km}$ de radio, un eNB por celda y un número variable de UE uniformemente distribuidos en las celdas. Los UE se desplazan entre las celdas siguiendo un camino aleatorio en un modelo de macrocelda urbano según lo definen Piro et al. (2011a). En este escenario cada UE tiene acceso a un flujo de IPTV unicast, un flujo de VoIP y un flujo de datos BE. A cada flujo se le asignó la configuración de los parámetros de calidad de servicio necesarios para satisfacer los requisitos de calidad. La simulación de este escenario permitió la evaluación del retardo, la pérdida de paquetes y el throughput frente a variaciones en la cantidad de terminales móviles conectados a la red elegidos de un rango de 5 a $35 \mathrm{UE}$, para diferente velocidad de movilidad de los usuarios 
(3 y $30 \mathrm{~km} / \mathrm{h}$ ), con diferentes tasas de codificación de los flujos de IPTV (242 kbps y $440 \mathrm{kbps}$ ) y variación de los algoritmos de planificación de paquetes en la red LTE (PF, M-LWDF y EXP/PF). Cabe señalar que en entornos de las redes móviles e inalámbricas las técnicas usadas para la codificación del video es H.264/MPEG-4 AVC y para la codificación del audio es MPEG-4 audio, debido a que con estas se logran las tasas de bits más bajas (128, 242 y $440 \mathrm{kbps})$ para afrontar las limitaciones de la velocidad de transmisión de este tipo de redes (Mcdonagh et al., 2011; 3GPP, 2012b).

Figura 1. Escenario de red LTE multicelda/multiusuario

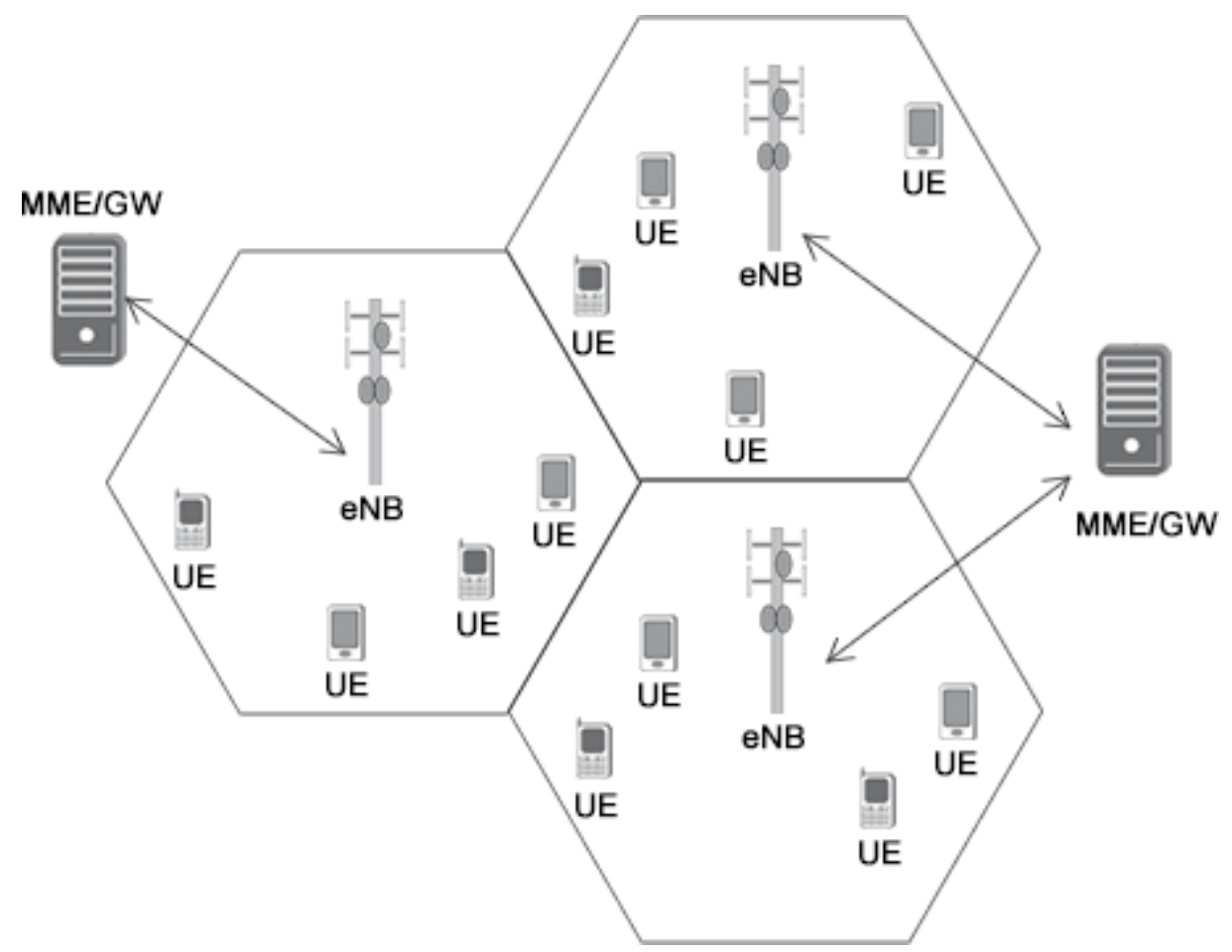

Fuente: presentación propia de los autores

\subsection{Construcción del escenario de simulación}

LTE-Sim abarca varios aspectos de las redes LTE, que incluye tanto la red de acceso Evolved-Universal Terrestrial Radio Access Network (E-UTRAN) como el núcleo Evolved Packet Core (EPC). Específicamente, fueron modelados tres tipos de nodos de la red LTE: UE, eNB y Mobility Management Entity/ Gateway (MME/GW), y soporta entornos unicelda y multicelda, gestión de 
calidad de servicio, movilidad del usuario (velocidad y dirección de desplazamiento) y procesos de handover (Piro et al., 2011a). También implementa varios generadores de tráfico en la capa de aplicación y soporta la gestión de RB. En LTE-Sim se programaron algoritmos de planificación de tráfico bien conocidos (PF, M-LWDF y EXP/PF); el esquema Adaptive Modulation and Coding (AMC); la retroalimentación calidad del canal CQI; las técnicas de reutilización de frecuencia; las pérdidas de propagación, y los modelos para la capa PHY (Piro et al., 2011a).

En LTE-Sim, cuando se establece un flujo de paquetes, se activa un portador $\mathrm{RB}$ dedicado entre el eNB y el UE tanto para el UL como para el DL (Piro $e t$ al., 2011a). Además, para cada UE y eNB se activa más de un RB con soporte a calidad de servicio. El modelo de calidad de servicio desarrollado en LTE-Sim está conformado por un clasificador de paquetes IP que permite mapear los paquetes procedentes de la capa IP a un RB específico. La clasificación de paquetes se realiza utilizando un filtro de paquetes basado en las direcciones IP de origen y destino, los puertos del emisor y del receptor y el tipo de protocolo de transporte (Piro et al., 2011a). En cada RB se define la configuración de los parámetros de calidad de servicio para el flujo de paquetes. Los parámetros de calidad de servicio que pueden configurarse en LTE-Sim son el QCI y el parámetro de asignación y retención de prioridad en los modos preemption flow capability y preemption flow vulnerability, Guaranteed Bit Rate (GBR) y Maximum Bit Rate (MBR) (Piro et al., 2011a).

\subsubsection{Modelo de red LTE para la simulación del servicio de IPTV}

El escenario de simulación corresponde a un modelo de red LTE de 9 macroceldas urbanas con un radio de $1 \mathrm{~km}$ y un clúster de 4 celdas, frecuencia de portadora de $2 \mathrm{GHz}$ en modo Frequency Division Duplex (FDD), canales en DL de $10 \mathrm{MHz}, 14$ símbolos por Transmission Time Interval (TTI), longitud de la subtrama de $1 \mathrm{~ms}, 12$ subportadoras por $\mathrm{RB}, 12 \mathrm{kHz}$ de separación entre subportadoras, potencia de transmisión de $43 \mathrm{dBm}$ y 2 antenas de transmisión en el eNB, con los esquemas de modulación QPSK, 16QAM y 64QAM, y $10 \%$ en Block Error Rate (BLER). El reporte del CQI lo hace el UE periódicamente en modo full bandwidth. Esta configuración se basa en los trabajos presentados por Piro et al. (2011a y 2011b). En la tabla 1 se presenta el resumen de los parámetros configurados para la simulación del modelo de red LTE. 
Tabla 1. Parámetros de entrada a para la simulación del modelo red LTE

\begin{tabular}{|l|l|l|}
\hline \multicolumn{1}{|c|}{ Parámetro } & \multicolumn{1}{c|}{ Valor } \\
\hline \multirow{5}{*}{ Capa PHY } & Frecuencia de portadora (Carrier Frecuency) & $2 \mathrm{GHz}$ \\
\cline { 2 - 3 } & Ancho de banda para el DL (Bandwidth) & $10 \mathrm{MHz}$ \\
\cline { 2 - 3 } & Símbolos por TTI (Symbol for TTI) & 14 \\
\cline { 2 - 3 } & Longitud de la subtrama (Subframe length) & $1 \mathrm{~ms}$ \\
\cline { 2 - 3 } & Subportadoras por RB (Subcarries per RB) & 12 \\
\cline { 2 - 3 } & Separación entre subportadoras (Subcarrier spacing) & $15 \mathrm{kHz}$ \\
\cline { 2 - 3 } & Potencia de transmisión en el eNB & $43 \mathrm{dBm}$ \\
\cline { 2 - 3 } & Número de antenas de transmisión en el eNB & 2 \\
\cline { 2 - 3 } & Esquema de modulación & QPSK, 16QAM y 64QAM \\
\cline { 2 - 3 } & Tasa de error de bloque (BLER) & $10 \%$ \\
\hline \multirow{5}{*}{ Overbead } & RTP/UDP/IP con compresión ROCH & 3 bytes \\
\cline { 2 - 3 } & MAC y RLC & 5 bytes \\
\cline { 2 - 3 } & PDCP & 2 bytes \\
\cline { 2 - 3 } & CRC & 3 bytes \\
\cline { 2 - 3 } & L1/L2 & 3 símbolos \\
\hline \multirow{5}{*}{ Usuarios } & Diámetro de la celda & $1 \mathrm{~km}$ \\
\cline { 2 - 3 } & Clúster & 4 \\
\hline \multirow{5}{*}{ RLC ARQ } & Número de retransmisiones para la activación & 5 \\
\hline CQI & Esquema de reporte & Full bandwidth, periódico \\
\hline & Número de usuarios/Celda & $5,15,25 \mathrm{y} 35$ \\
\cline { 2 - 3 } & Movilidad & Dirección aleatoria $30 \mathrm{~km} / \mathrm{h}$ \\
\hline
\end{tabular}

Fuente: presentación propia de los autores

\subsubsection{Modelo de tráfico para la simulación de las aplicaciones}

Los paquetes transportados por un $\mathrm{RB}$ dedicado se producen en la capa de aplicación de LTE-Sim mediante cuatro generadores de tráfico independientes: basado en trazas (trace-based) para la simulación de IPTV, VoIP, Constant Bit Rate (CBR) y buffer infinito. En LTE-Sim, el proceso de transmisión de paquetes se inicia con la generación de los paquetes de cada aplicación, los cuales pasan a través de la pila de protocolos de plano de usuario donde se adicionan los encabezados de protocolos (Piro et al., 2011a). Luego el paquete se pone en cola en la capa Media Access Control (MAC) y se asocia con un portador de radio utilizando las funcionalidades del clasificador IP (Piro et al., 2011a). Con esto, el paquete queda listo para ser enviado por el canal basándose en las decisiones del algo- 
ritmo de planificación de paquetes configurado en el eNB (Piro et al., 2011a). Cuando el nodo de destino recibe los paquetes desde el canal de transmisión, se eliminan los encabezados conforme atraviesa la pila de protocolos del plano de usuario hasta que el paquete es finalmente entregado al disipador de aplicación encargado de procesar el paquete (Piro et al., 2011a).

En el escenario planteado cada uno de los UE tiene acceso a un flujo de IPTV, un flujo de VoIP y un flujo de datos BE. En la herramienta de simulación los flujos de tráfico fueron modelados de la siguiente manera:

- El flujo de IPTV de VoD fue simulado mediante la aplicación trace-based de LTE-Sim. La traza de tráfico se creó a partir de las secuencias de prueba de video foreman.yuv (Asu, 2013), que corresponde a un video con 25 tramas por segundo, una resolución CIF de $352 \times 288$ y una formato YUV que fue repetido durante todo el tiempo de simulación (Piro et al., 2011a). La secuencia de video obtenida fue comprimida usando el estándar MPEG-4/H.264 con tasa de codificación promedio de $242 \mathrm{kbps}$ y $440 \mathrm{kbps}$ (Piro et al., 2011a).

- El flujo de VoIP fue modelado con cadena de Markov ON/OFF, donde el periodo $\mathrm{ON}$ está distribuido exponencialmente con un valor medio de $3 \mathrm{~s}, \mathrm{y}$ el periodo OFF tiene una función de densidad de probabilidad exponencial truncada con un límite superior de 6,9 s y un valor medio de 3 s (Chuah y Katz, 2002). Durante el periodo de ON, el origen envía paquetes con un tamaño de 20 bytes cada 20 ms para lograr una tasa de bits de $8 \mathrm{kbps}$, mientras que durante el periodo de OFF, la tasa de bits es 0 , debido a que se asume la presencia de un detector de actividad de voz (Piro et al., 2011a).

- El flujo BE corresponde a una aplicación de buffer infinito para modelar una fuente ideal que siempre tiene paquetes para enviar (Piro et al., 2011a).

\section{Análisis de resultados y discusión}

Los parámetros de desempeño evaluados son el retardo, la tasa de pérdida de paquetes y el throughput para cada una de las aplicaciones (IPTV, VoIP y datos BE). En este sentido, en las figuras 2 y 3 se presentan los resultados de la simulación para el retardo de los flujos de paquetes de IPTV y VoIP, respectivamente. Como se puede observar, el retardo para los dos servicios aumenta cuando crecen el número de usuarios conectados a la red y la velocidad de desplazamiento. También se puede apreciar que si la tasa de codificación de los flujos de video es más alta, el retardo IPTV y VoIP aumenta, puesto que se incrementa el tráfico de video y, por consiguiente, los paquetes tardan más tiempo en las colas de los planificadores de los eNB antes de ser transmitidos por la red LTE. 
Figura 2. Retardo de flujos IPTV con tasa de codificación de a) 242 kbps y b) 440 kbps

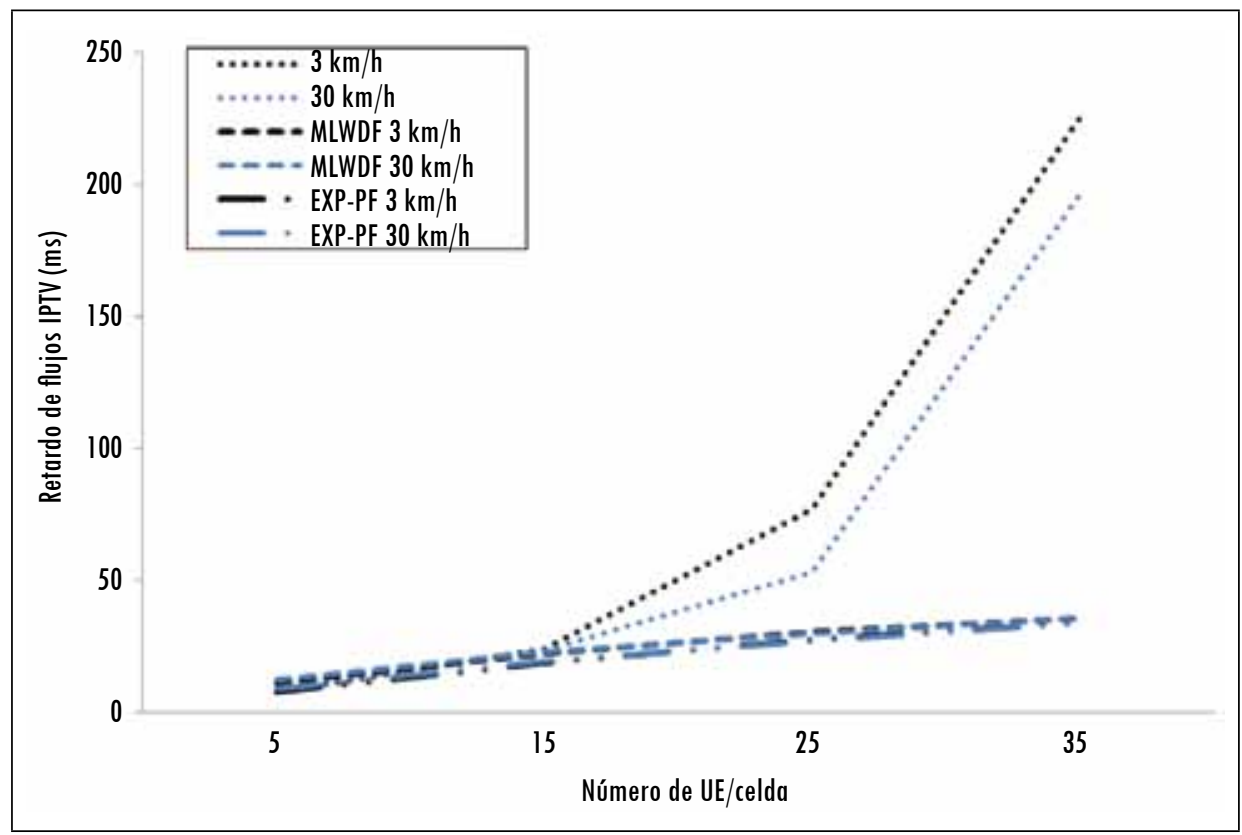

a)

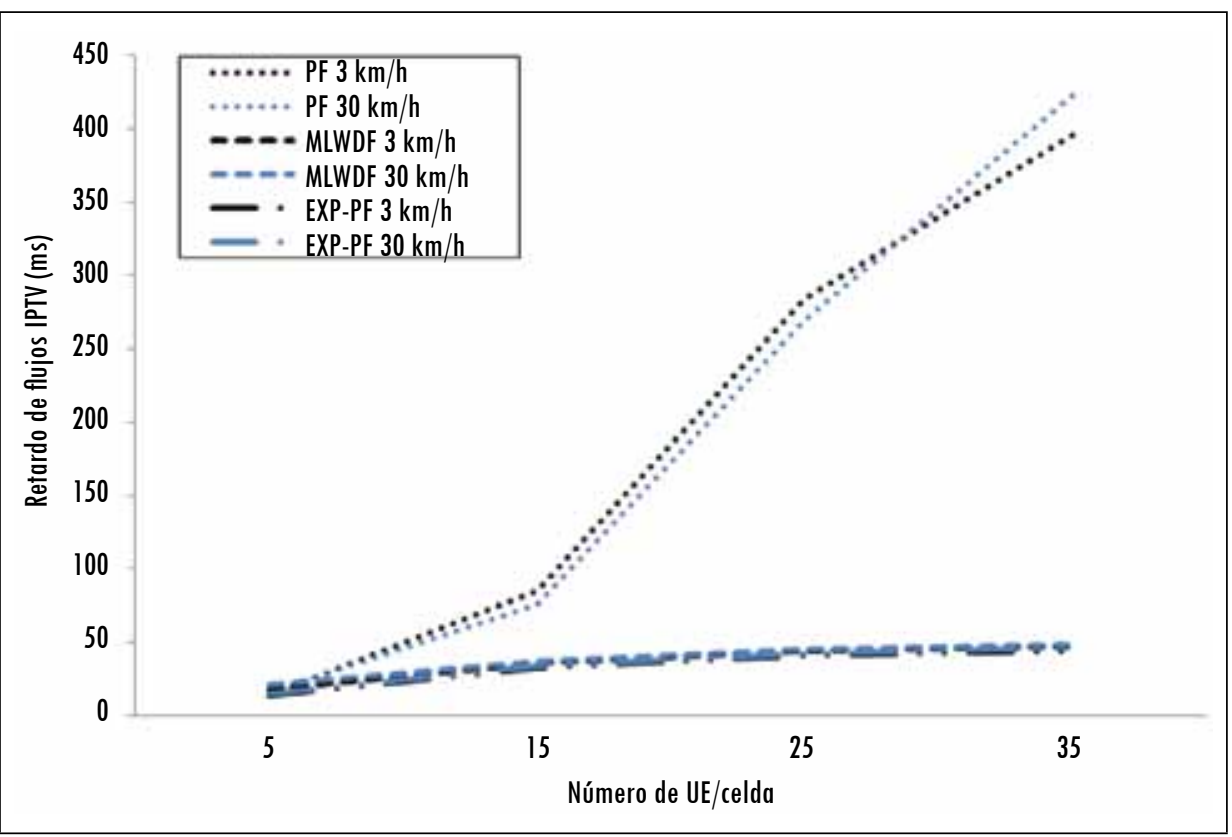

b)

Fuente: presentación propia de los autores 
En cuanto al efecto de los algoritmos de planificación de paquetes, el mejor comportamiento del retardo para los flujos de IPTV y VoIP se obtuvo cuando se configuran los planificadores M-LWDF y EXP/PF, pues se logra mantener un retardo por debajo del requisito de calidad de servicio para estas aplicaciones, cuyo valor máximo permitido es de $100 \mathrm{~ms}$ para IPTV (ITU-T, 2008; ITU-T, 2011) y $150 \mathrm{~ms}$ para VoIP (ITU-T, 2003). En las figuras 2 y 3 se puede ver que con estos algoritmos el promedio del retardo para IPTV es de $30 \mathrm{~ms}$ y para los flujos de VoIP el retardo es de 2,26 ms. Si se configura el algoritmo PF, el retardo IPTV se incrementa desde $7 \mathrm{~ms}$ para $5 \mathrm{UE}$ hasta los $400 \mathrm{~ms}$ para $35 \mathrm{UE}$ (figura 2). Esto significa que PF no es recomendable para mantener un bajo retardo en los flujos de IPTV ante el crecimiento del número de UE, ya que PF soporta sus decisiones de planificación de tráfico en las condiciones del canal y el throughput de la red, insuficientes para soportar aplicaciones de video, debido a los estrictos requisitos de retardo. Sin embargo, con el algoritmo PF, el retardo de los flujos de VoIP se mantiene en promedio entre 1,76 ms (figura 3).

En las figuras 4 y 5 se muestran los resultados de las simulaciones para la tasa pérdida de paquetes de los flujos de IPTV y VoIP. Para los dos servicios, la razón de pérdida de paquetes aumenta con la velocidad de desplazamiento de los UE, puesto que la calidad del canal de comunicaciones inalámbrico disminuye y el procedimiento de adaptación de enlace se deteriora a altas velocidades. Adicionalmente, se tiene que si la tasa de codificación de video aumenta, la razón de pérdida de paquetes crece, debido a que los planificadores de tráfico tienen que procesar una mayor cantidad de paquetes de video, lo cual desborda las colas de tráfico. También se observa que cuando se utilizan los planificadores M-LWDF y EXP/PF, la razón de pérdida de paquetes es menor que cuando se usa el algoritmo PF.

Además, para los planificadores M-LWDF y EXP/PF, la razón de pérdida de paquetes se incrementa con el número de UE, porque un alto número de flujos en tiempo real generan que la probabilidad de descarte de paquetes sea más grande por la expiración del tiempo de espera de transmisión. Es de tener en cuenta que la razón de pérdida de paquetes en redes móviles puede ser superior al $1 \%$ (Mcdonagh et al., 2011) y estos algoritmos logran mantener la razón de pérdida de paquetes cercana a este valor de referencia cuando el número de usuarios es bajo y la velocidad de desplazamiento es menor. Al utilizarse el planificador PF, la razón de pérdida de paquetes aumenta en mayor medida con el número de UE conectados a la red, ya que este algoritmo no está diseñado para manejar un alto volumen de tráfico de aplicaciones en tiempo real y, por ello, descarta más paquetes que los planificadores M-LWDF y EXP/PF. 
Figura 3. Retardo de flujos VoIP cuando la tasa de codificación de video es de a) 242 kbps y b) 440 kbps

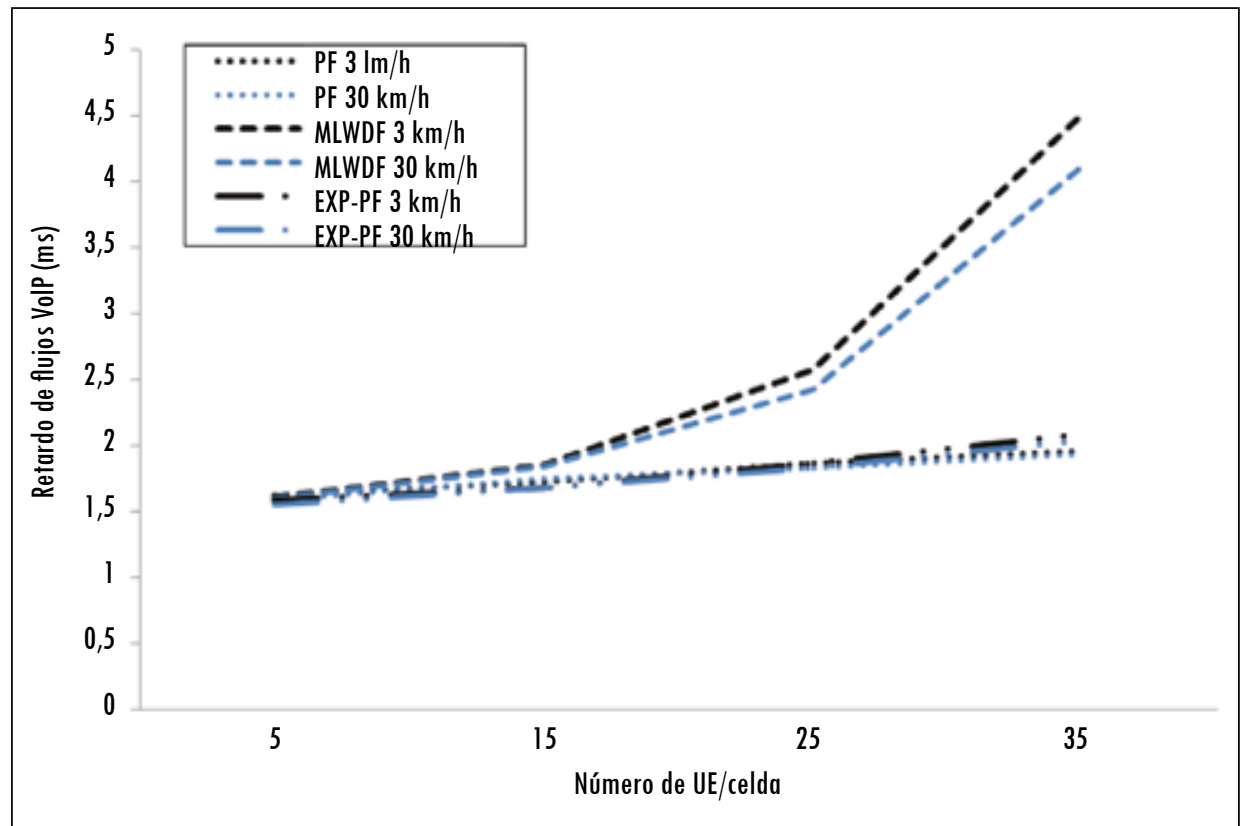

a)

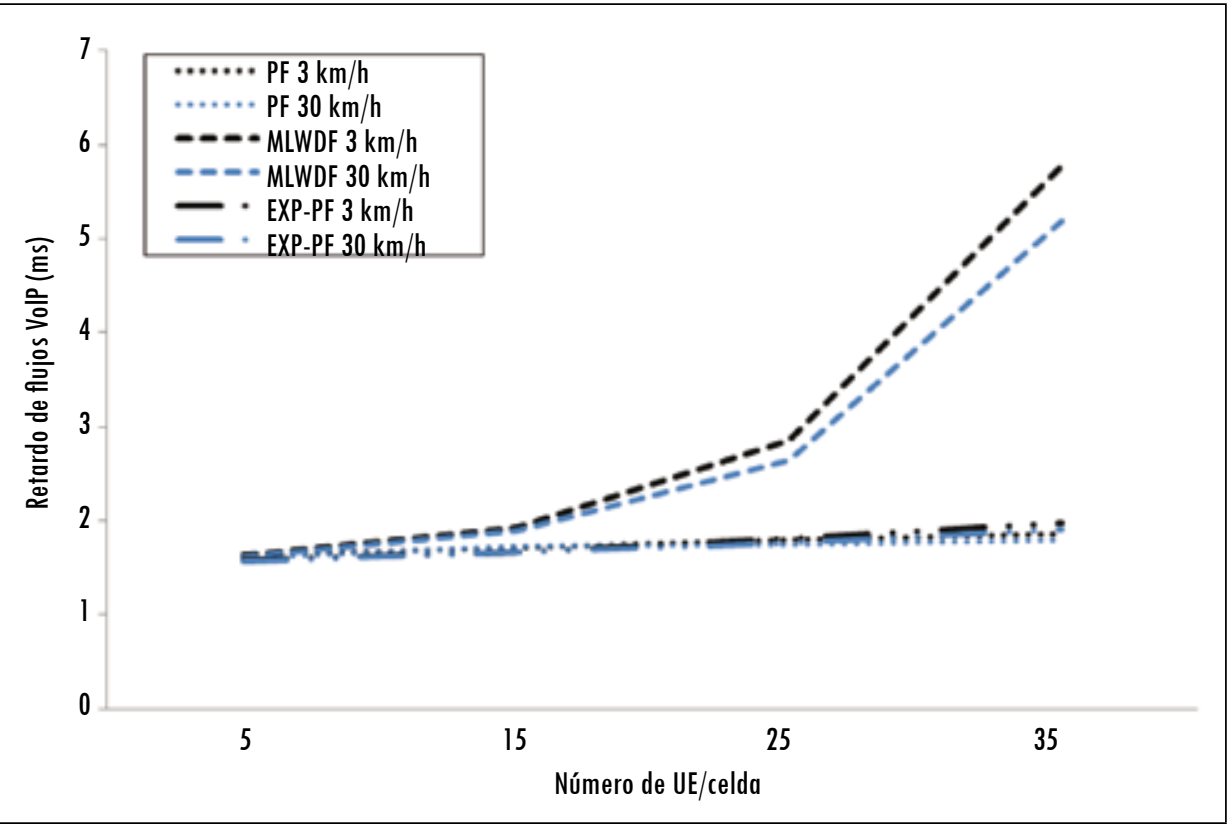

b)

Fuente: presentación propia de los autores 
Figura 4. Razón de pérdida de paquetes de flujos IPTV con tasa de codificación de a) 242 kbps y b) 440 kbps

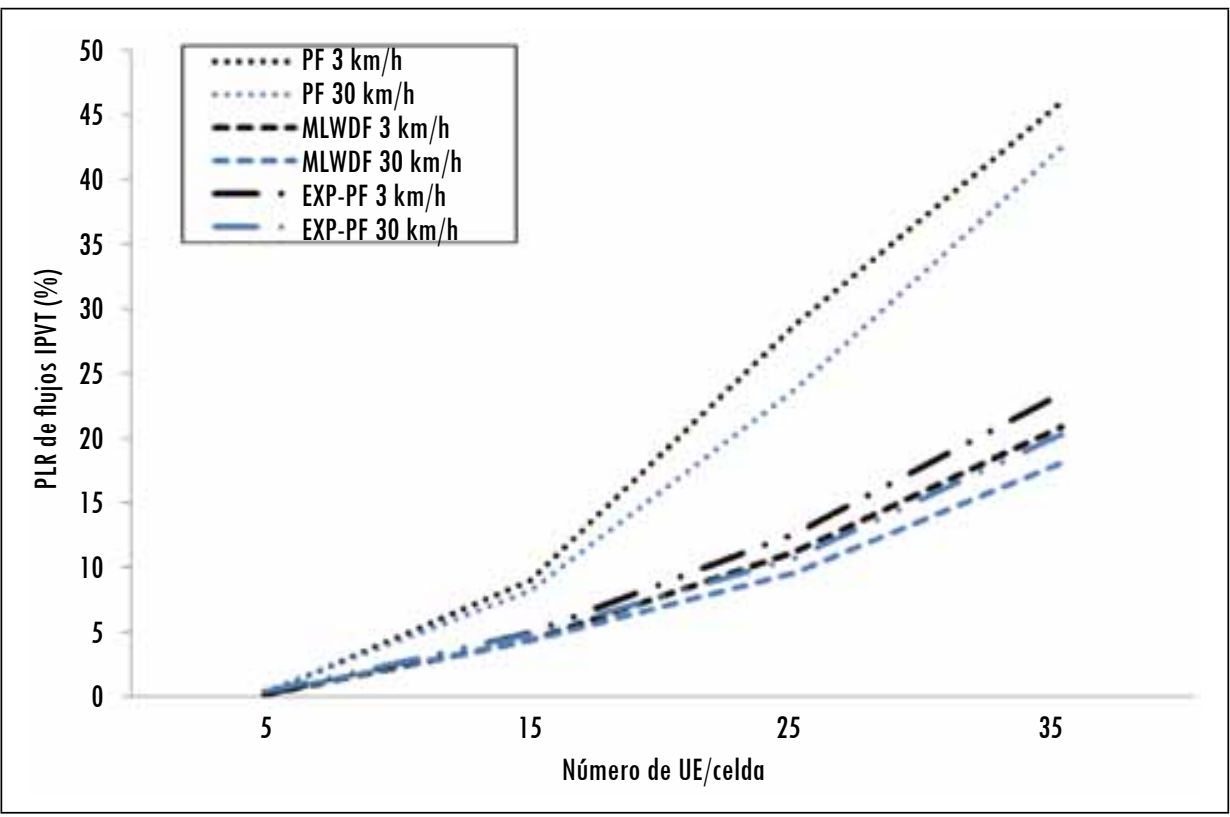

a)

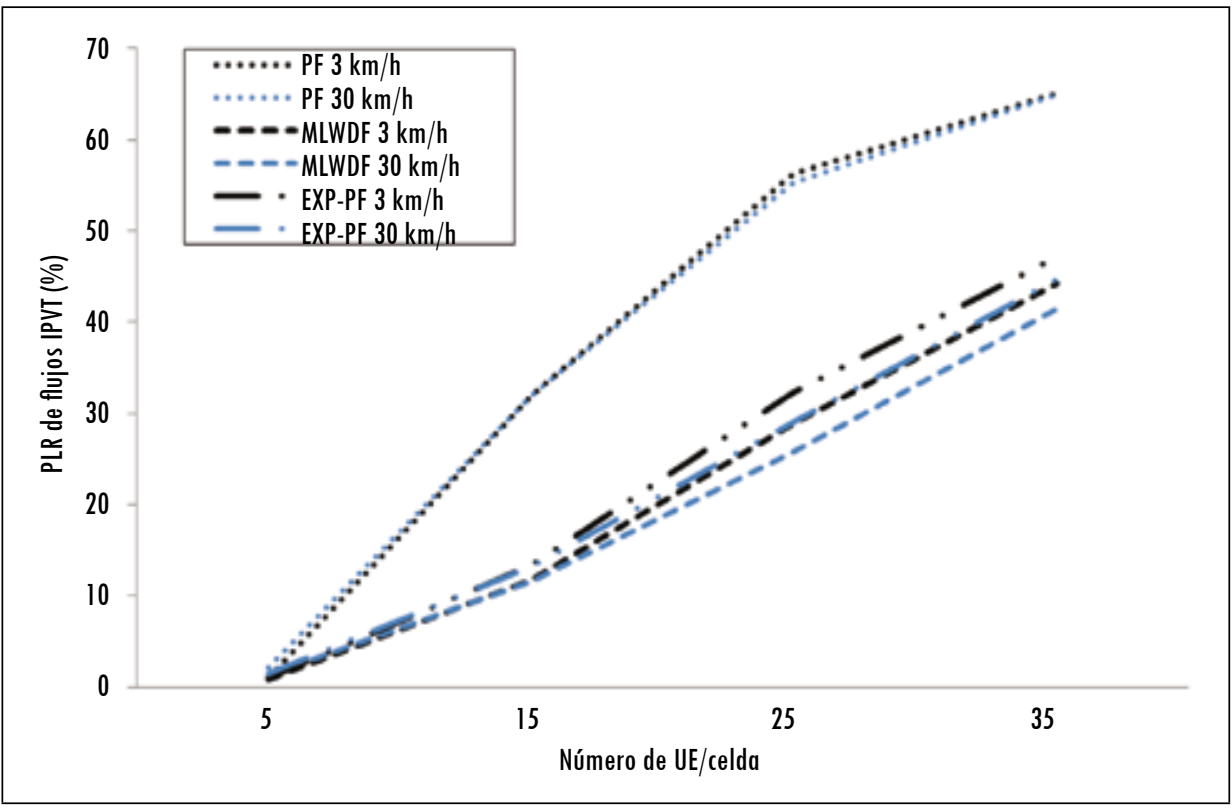

b)

Fuente: presentación propia de los autores

Ing. Univ. Bogotá (Colombia), 18 (2): 393-417, julio-diciembre de 2014 
Figura 5. Razón de pérdida de paquetes de flujos VoIP cuando la tasa de codificación de video es de a) 242 kbps y b) 440 kbps

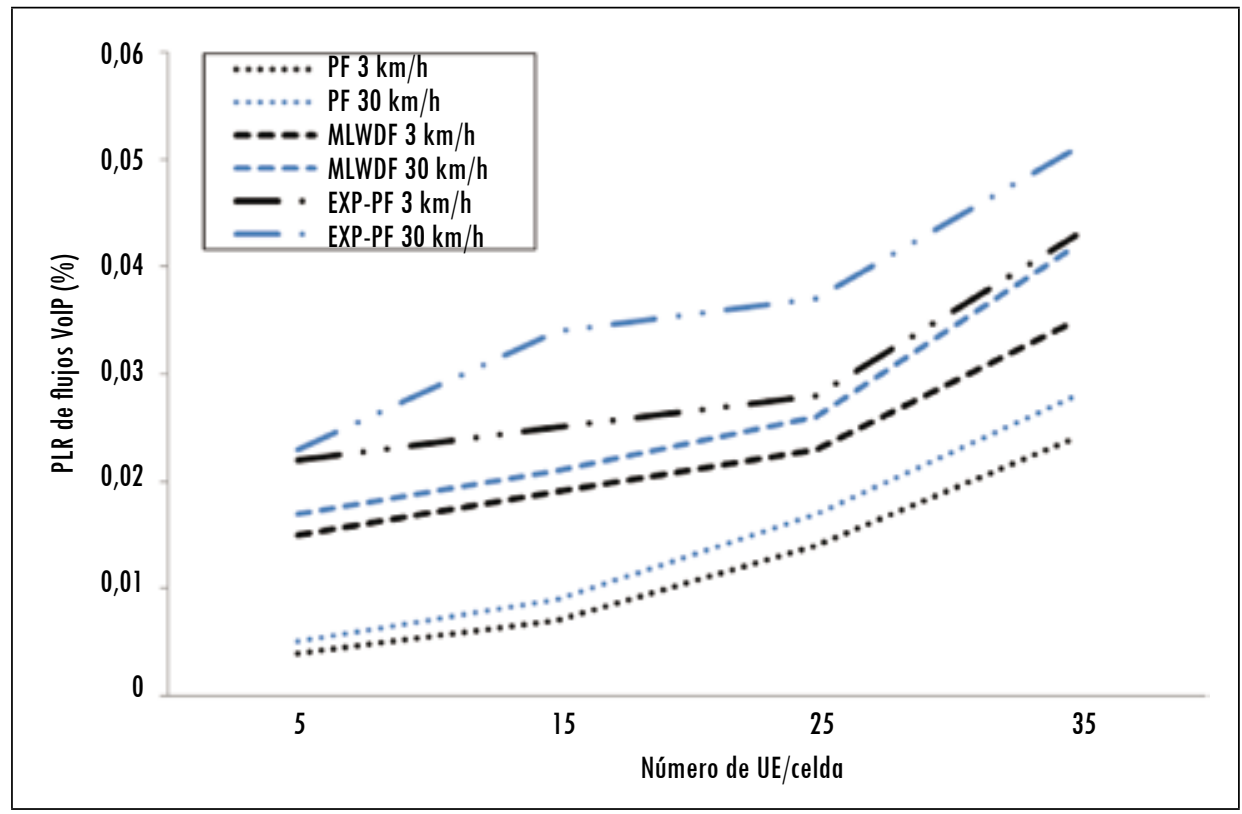

a)

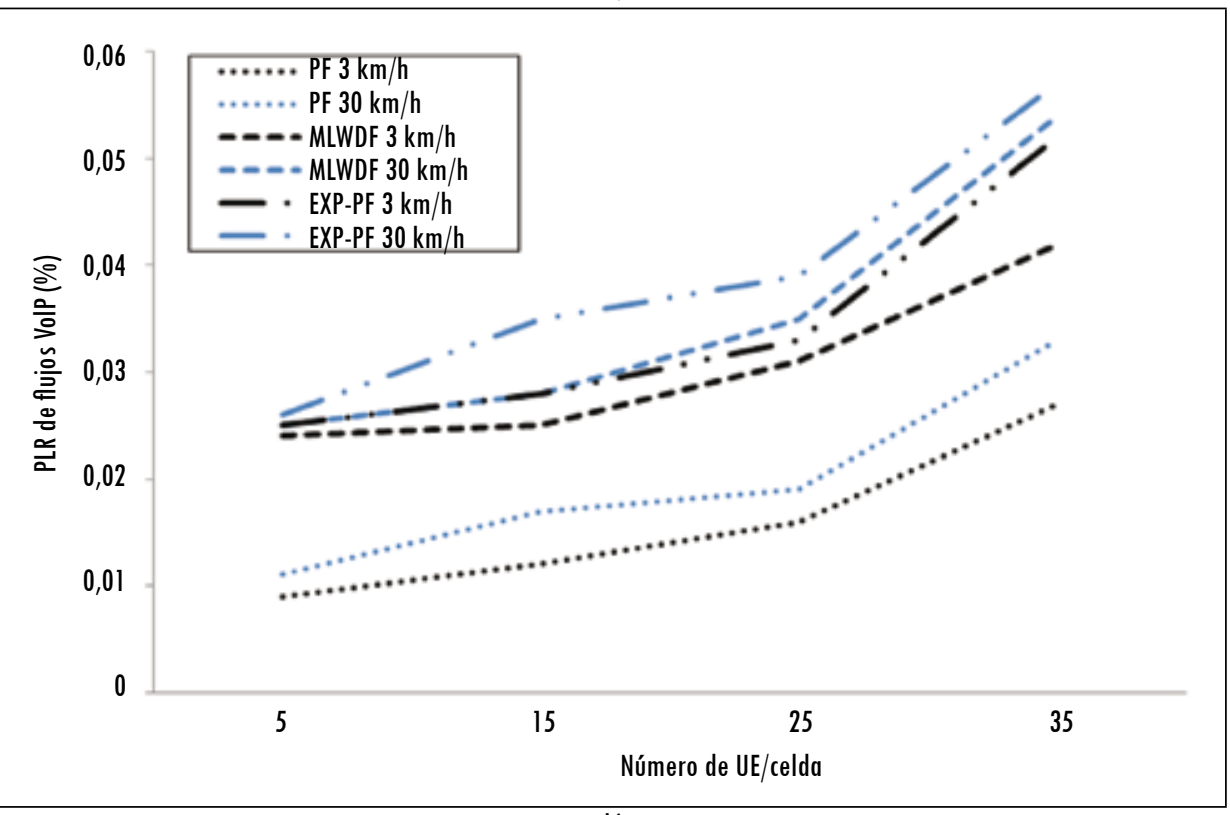

b)

Fuente: presentación propia de los autores 
En lo que respecta al throughput de los flujos de paquetes de IPTV y VoIP, se observa que este parámetro disminuye cada vez que el número de UE aumenta. Esta situación se debe principalmente a que la razón de pérdida de paquetes aumenta en la medida en que crecen los UE conectados a la red tal, y como se puede ver en las figuras 6 y 7 . En consecuencia, el crecimiento de la razón de pérdida de paquetes se traduce en una menor transferencia efectiva de paquetes por la red. El mayor throughput para los flujos de IPTV y VoIP se obtuvo con los algoritmos de planificación M-LWDF y EXP/PF, mientras que con PF el throughput es el más bajo. Igualmente, se puede apreciar que si la tasa de codificación de video aumenta, el throughput de los flujos de IPTV disminuye, debido a que los paquetes son descartados en las colas de los planificadores y, por ende, en la red LTE circula menos tráfico asociado con este servicio.

Del mismo modo, se evaluó el desempeño de la aplicación de transferencia de datos $\mathrm{BE}$, donde el throughput disminuye cuando la velocidad de desplazamiento aumenta, debido a que la calidad del canal empeora con la velocidad. Con las estrategias de planificación de paquetes evaluadas, los flujos de paquetes BE recibieron el mismo trato; por tal razón no se observan diferencias significativas en la variación de throughput y en la razón de pérdidas de paquetes. En la figura 8 se presenta la razón de pérdida de paquetes, y en la figura 9, el throughput de los flujos de paquetes BE.

Por último, la figura 10 muestra la eficiencia espectral en la red LTE, la cual se expresa como el throughput total de todos los usuarios dividido por el ancho de banda disponible. Como se ilustra en la figura 10, el impacto de cada uno de los algoritmos de planificación de tráfico sobre esta variable es diferente. Precisamente, cuando el número de usuarios en la celda aumenta, los planificadores M-LWDF y EXP/PF tratan de garantizar la calidad de servicio para un gran número de flujos, con un impacto negativo en la eficiencia del sistema; sin embargo, la eficiencia espectral está por encima de la lograda con el algoritmo PF. También se tiene que la eficiencia espectral es mayor cuando la velocidad de desplazamiento disminuye, puesto que los UE perciben una mejor calidad del canal y no se descartan paquetes por errores en la transmisión por el canal PHY.

Los resultados obtenidos en este trabajo, a partir de la evaluación del comportamiento de los principales parámetros de desempeño de la red, son similares a los encontrados en Ramli et al. (2009) y Piro et al., (2011a), lo cual significa que los resultados de las simulaciones realizadas son coherentes y, además, permitieron verificar que la arquitectura propuesta en Rueda y Ramos (2012) se puede implementar configurando la red LTE con el planificador de tráfico M-LWDF, el cual demostró ser el mejor algoritmo para mantener una entrega de contenidos de IPTV dentro de los objetivos de calidad de servicio. 
Figura 6. Throughput de flujos IPTV con tasa de codificación de a) 242 kbps y b) 440 kbps

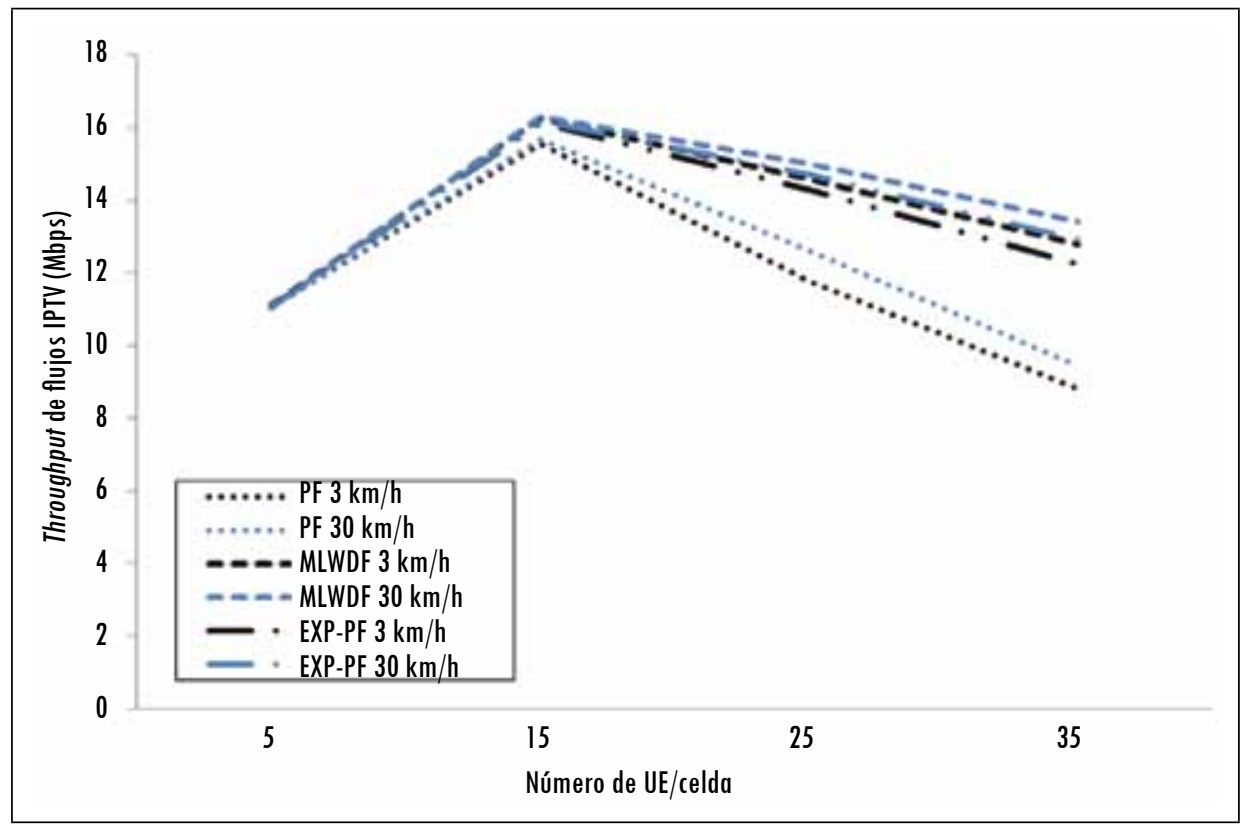

a)

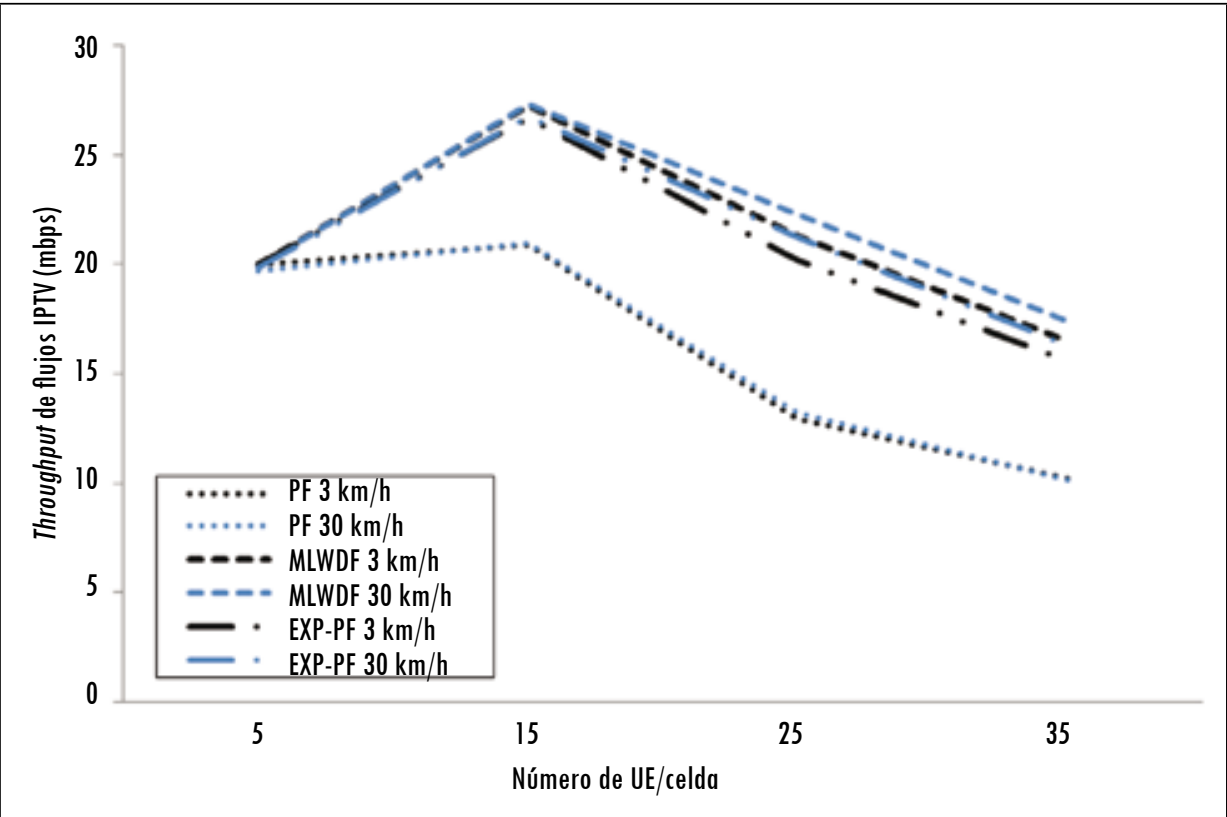

b)

Fuente: presentación propia de los autores 
Figura 7. Throughput de flujos VolP cuando la tasa de codificación de video es de a) 242 kbps y b) $440 \mathrm{kbps}$

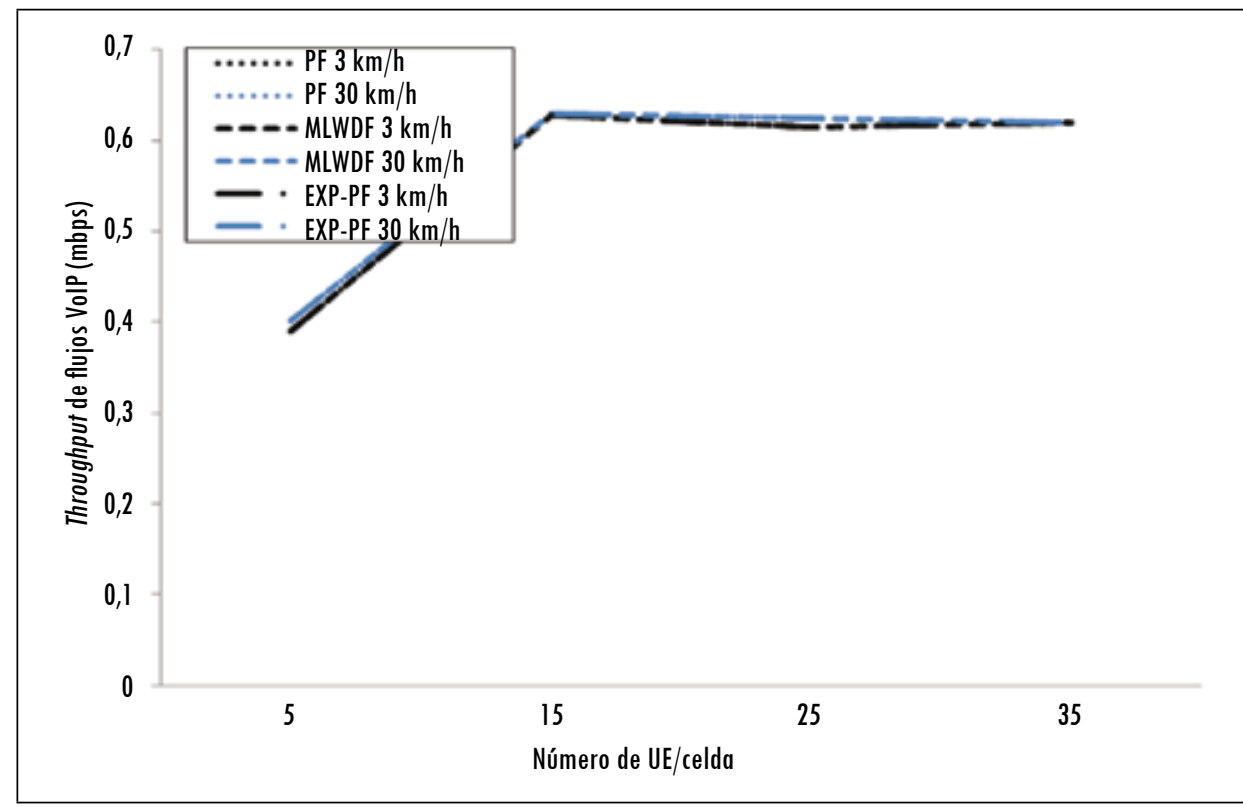

a)

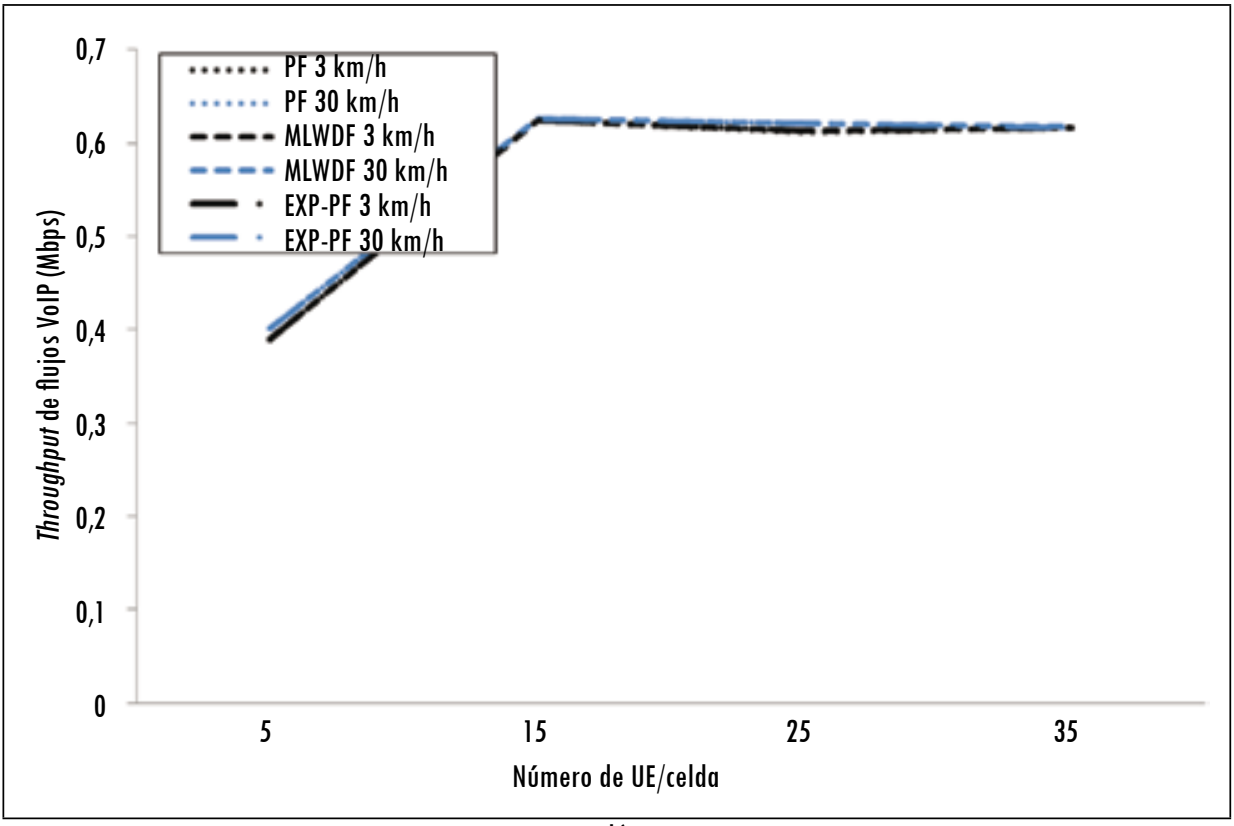

b)

Fuente: presentación propia de los autores 
Figura 8. Razón de pérdida de paquetes de flujos BE cuando la tasa de codificación de video es de a) 242 kbps y b) 440 kbps

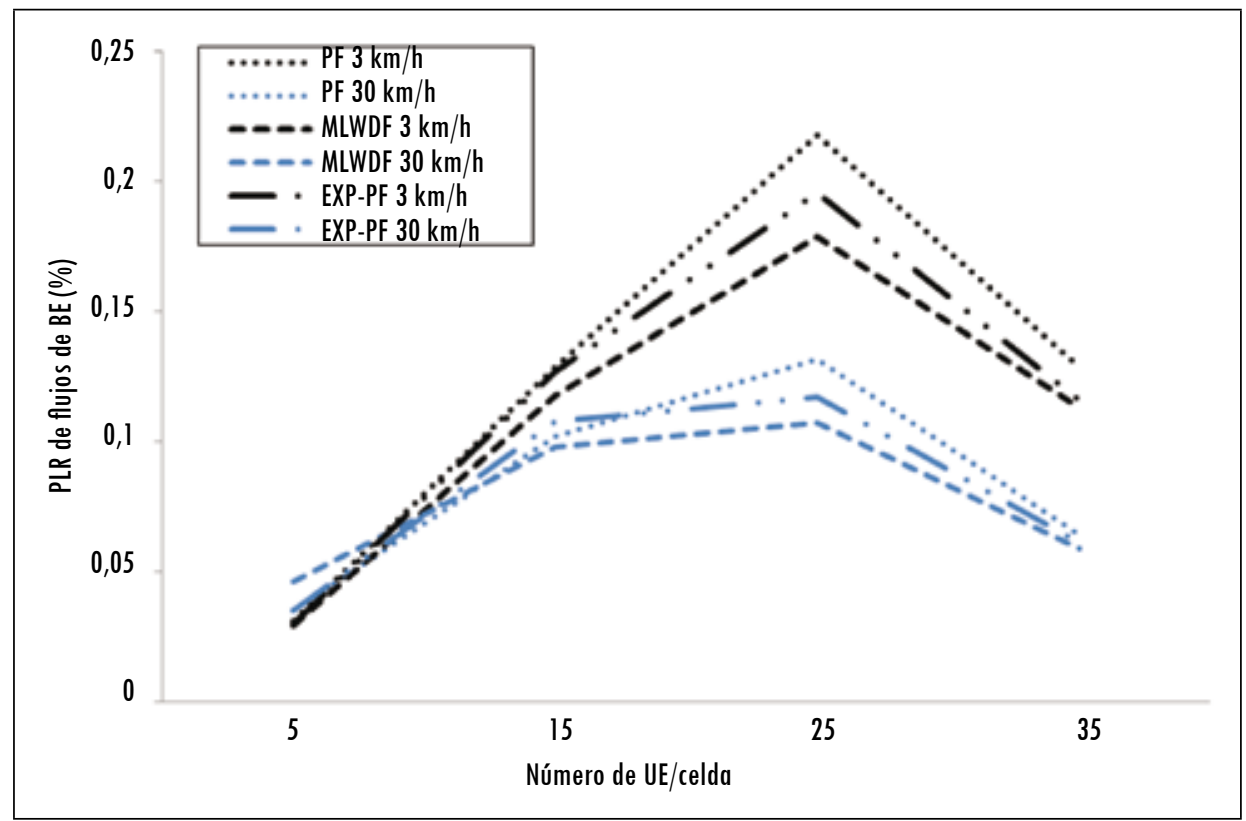

a)

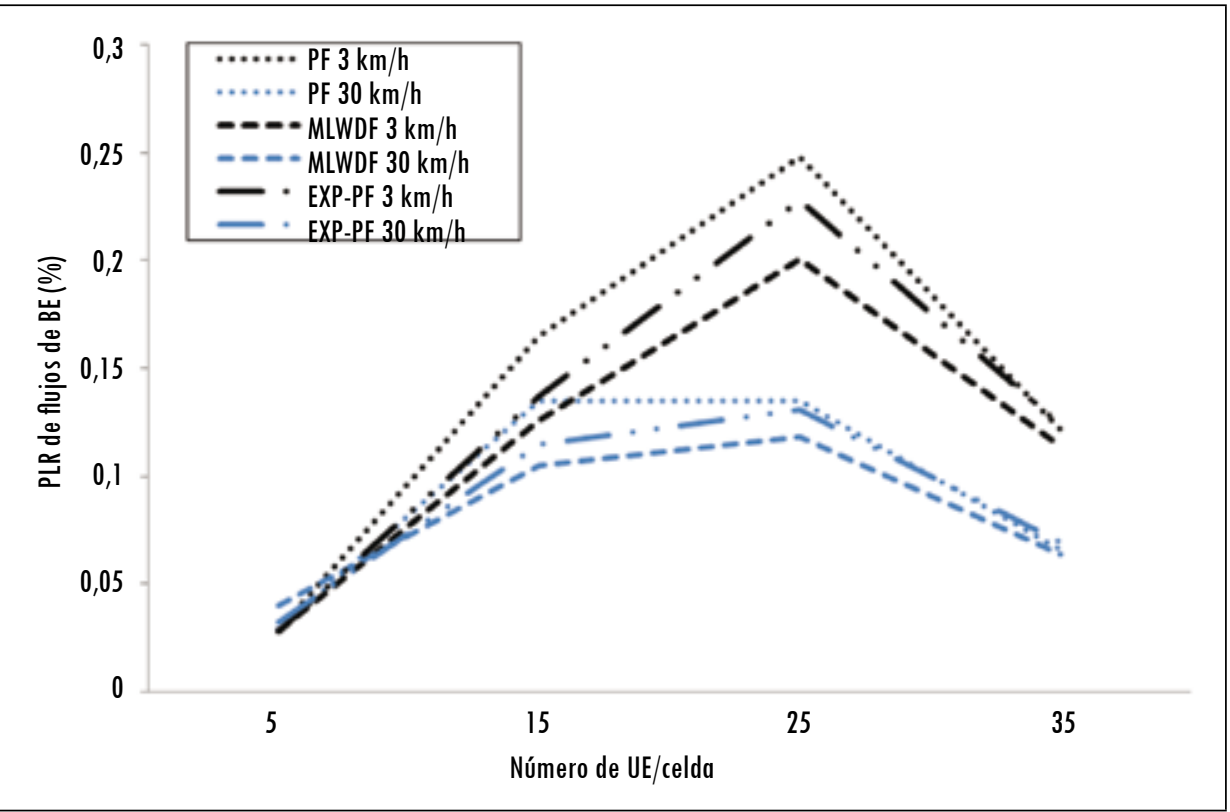

b)

Fuente: presentación propia de los autores 
Figura 9. Throughput de flujos BE cuando la tasa de codificación de video es de a) 242 kbps y

b) 440 kbps

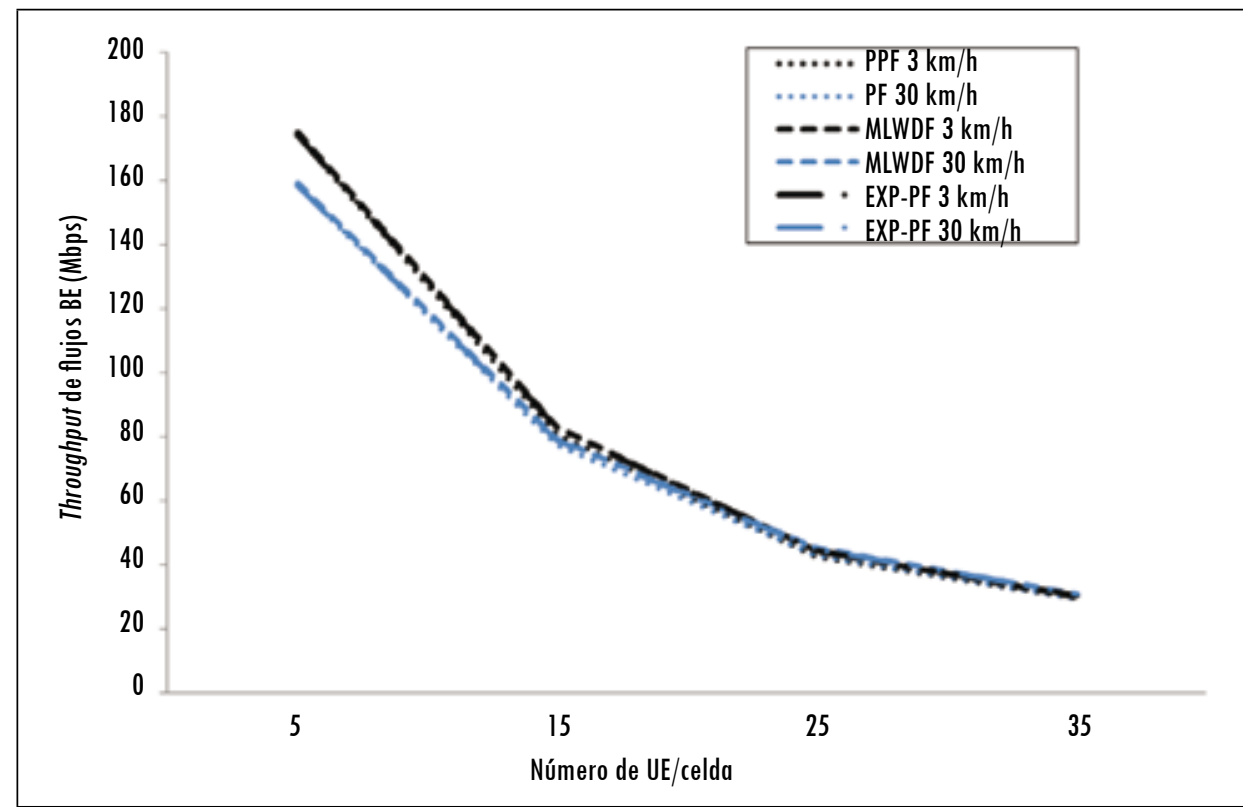

a)

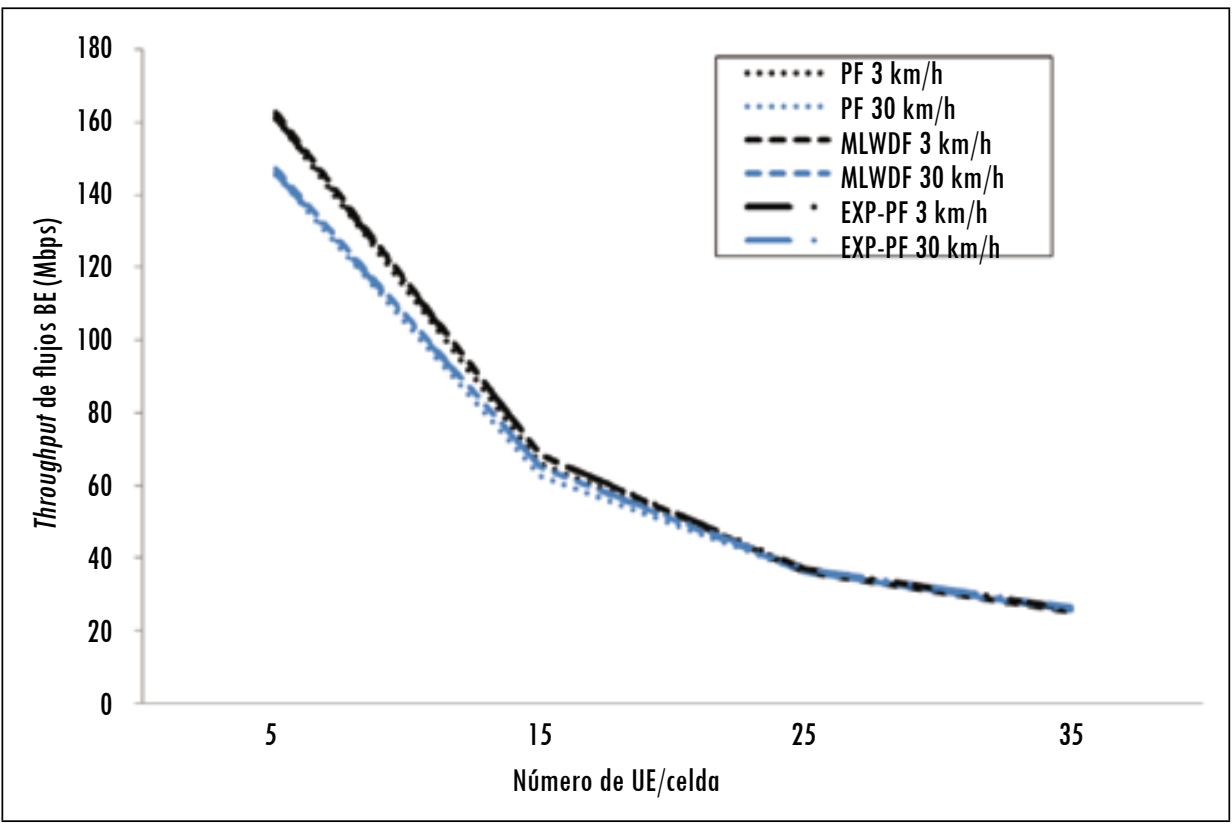

b)

Fuente: presentación propia de los autores 
Figura 10. Eficiencia espectral de la red LTE cuando la tasa de codificación de video es de a) 242 kbps y b) 440 kbps

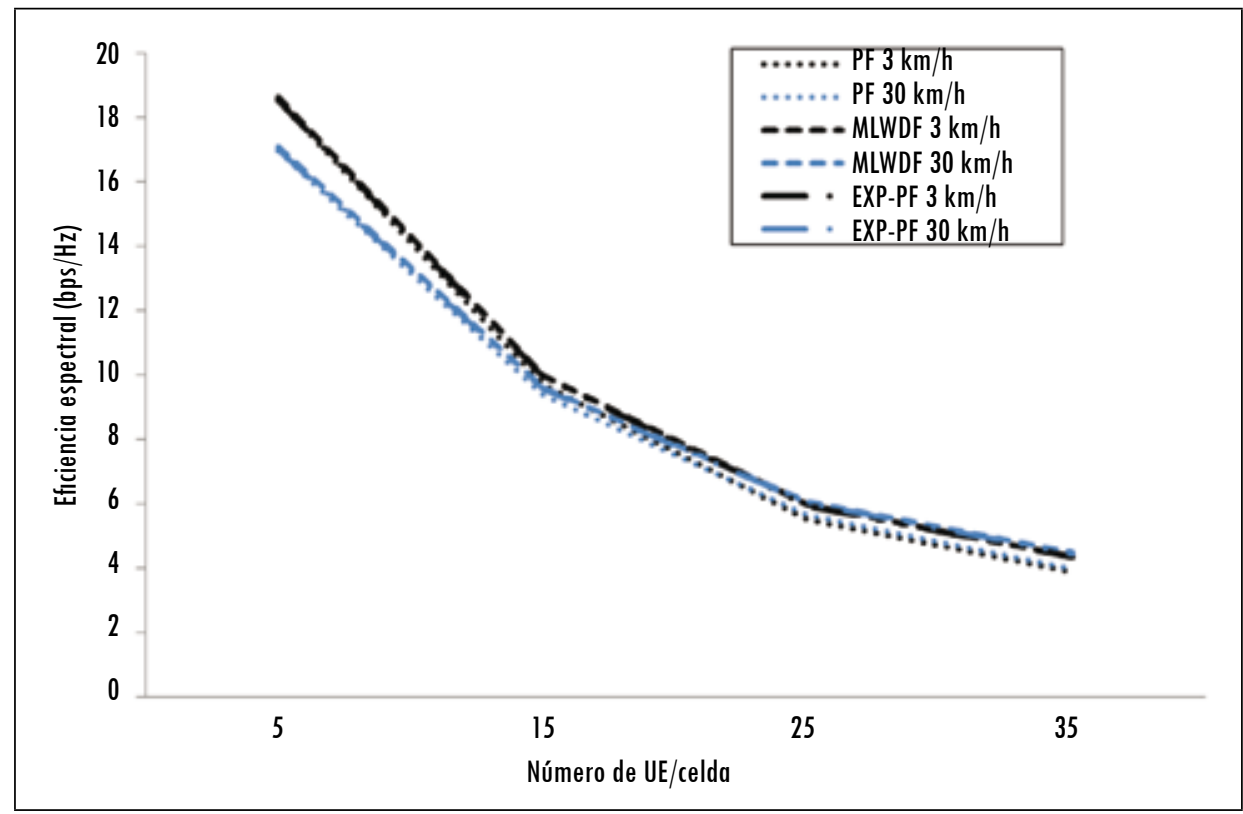

a)

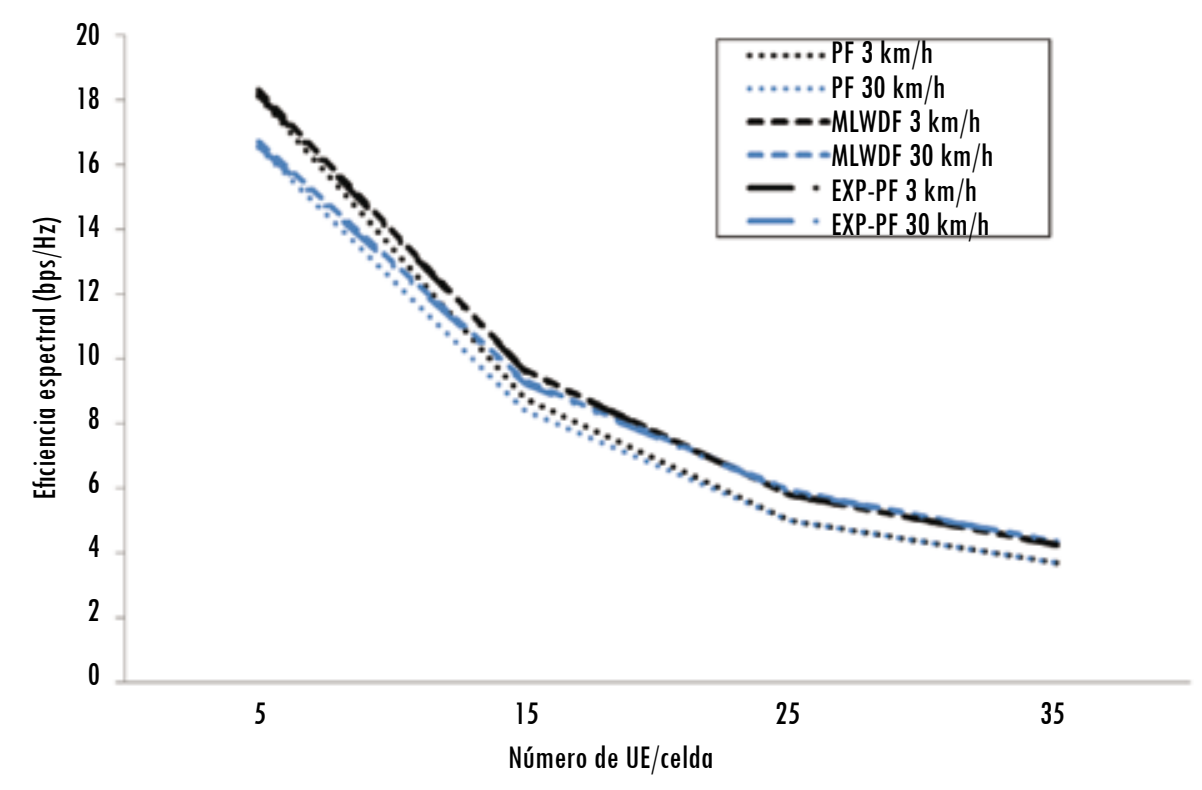

b)

Fuente: presentación propia de los autores 


\section{Conclusiones}

El servicio de conectividad IP proporcionado por las redes LTE soporta calidad de servicio, pues logra que los paquetes de datos de una determinada conexión puedan ser tratados de forma diferenciada para adaptarse a las necesidades de transmisión de cada uno de los servicios a los cuales pueden acceder los usuarios. Esto permite que las redes LTE soporten múltiples servicios con diferentes requisitos de desempeño y que garanticen a los usuarios una adecuada experiencia de uso de los servicios, y a los operadores móviles, una gestión eficiente de los recursos de red.

De los resultados de las simulaciones se puede inferir que el algoritmo de planificación de paquetes M-LWDF soporta la entrega del servicio de IPTV móvil, al garantizar el mejor comportamiento de los parámetros de desempeño (retardo, razón de pérdida de paquetes y throughput) ante el crecimiento en el número de usuarios y variación en la velocidad de desplazamiento. Este comportamiento se justifica, puesto que para cada flujo en tiempo real, M-LWDF define la probabilidad de que el máximo retardo del primer paquete que se va a transmitir en la cola supere el umbral del retardo. Para dar prioridad a flujos en tiempo real con el mayor retardo para los primeros paquetes de la cola y las mejores condiciones de canal, se define una métrica basada en la tasa media de transmisión de datos y la tasa de datos instantánea disponible de la UE receptor y el retado. En su lugar, para flujos en tiempo no real, la métrica considerada es la del algoritmo PF, cuyo objetivo es maximizar el throughput total de la red y garantizar la equidad entre los flujos. En la implementación del planificador M-LWDF, los paquetes pertenecientes a un flujo en tiempo real se borran de la cola de la capa MAC si no se transmiten antes que expire el su plazo para transmitirse. Esta operación es necesaria para evitar el desperdicio de ancho de banda.

\section{Referencias}

3GPP TS 23.107 V11.0.0. Quality of Service (QoS) concept and architecture. 3GPP. 2012a.

3GPP TS 26.234 V10.4.0. Transparent end-to-end Packet-switched Streaming Service (PSS); Protocols and codecs (Release 11). 3GPP. 2012b.ABU-LEBDEH, M.; BELQASMI, F.; GLITHO, R. 3GPP 4G evolved packet core-based system architecture for QoS-enabled mobile video surveillance applications. 2012 Third International Conference on the Network of the Future (NOF). Gammarth, 21-23 de noviembre 2012. [Gammarth]: IEEE, 2012, pp. 1-6.

ASU. Video trace library [en línea]. < http://trace.eas.asu.edu > [consulta: 01-03-2013].

BALINT, C.; BUDURA, G. and MARZA, E. Scheduling techniques evaluation in LTE systems with mixed data traffic. 2010 9th International Symposium in Electronics and Telecommuni- 
cations (ISETC). Timisoara, 11-12 de noviembre 2010. [Timisoara]: IEEE, 2010, pp. 221-224.

BASUKALA, R.; RAMLI, H. A. M.; SANDRASEGARAN, K. and CHEN, L. Impact of CQI feedback rate/delay on scheduling video streaming services in LTE downlink. 2010 12th IEEE International Conference in Communication Technology (ICCT), Nanjing, 11-14 de noviembre 2010. [Nanjing]: IEEE, 2010, pp. 1349-1352.

CHENG, X. and MOHAPATRA, P. Quality-optimized downlink scheduling for video streaming applications in LTE networks. Global Communications Conference (GLOBECOM), Anaheim, 3-7 de diciembre 2012. [Anaheim]: IEEE, 2012, pp. 1914-1919.

CHUAH, C. and KATZ, R. H. Characterizing packet audio streams from Internet multimedia applications. 2002 IEEE International Conference on Communications (ICC 2002). IEEE, 2002, pp. 1199-1203.

EKSTROM, H. QoS control in the 3GPP Evolved Packet System. IEEE Communications Magazine. 2009, vol. 47, no. 2, pp. 76-83.

GAO, H.; OUYANG, Y.; HU, H. and KOUCHERYAVY, Y. A QoS-guaranteed resource scheduling algorithm in high-speed mobile convergence network. Wireless Communications and Networking Conference Workshops (WCNCW), Shanghai, 7-10 de abril 2013. [Shanghai]: IEEE, 2013, pp. 45-50.

KUMAR, U. and OYMAN, O. QoE evaluation for video streaming over eMBMS. 2013 International Conference on Computing, Networking and Communications (ICNC), San Diego, 28-31 de enero 2013. [San Diego]: IEEE, 2013, pp. 555-559.

MCDONAGH P.; VALLATI C.; PANDE A.; MOHAPATRA P.; PERRY P. and MINGOZZI E. Investigation of Scalable Video Delivery using H. 264 SVC on an LTE Network. 14th Wireless Personal Multimedia Communications Symposium (WPMC'11), Brest, 3-7 de octubre 2011. [Brest]: IEEE, 2011, pp. 1-5.

PIRO, G.; GRIECO, L.; BOGGIA, G.; CAPOZZI, F. and CAMARDA, P. Simulating LTE cellular systems: an open source framework. IEEE Transactions on Vebicular Technology. 2011a, vol. 60, no. 2, pp. 498-513.

PIRO, G.; GRIECO, L.; BOGGIA, G.; FORTUNA, R. and CAMARDA, P. Two-Level Downlink Scheduling for Real-Time Multimedia Services in LTE Networks. IEEE Transactions on Multimedia. 2011b, vol. 13, no. 5, pp. 1052-1065.

RAMLI, H. A. M.; BASUKALA, R. M.; SANDRASEGARAN, K. and PATACHAIANAND, R. Performance of well-known packet scheduling algorithms in the downlink 3GPP LTE system. 2009 IEEE 9th Malaysia International Conference in Communications (MICC), Kuala Lumpur, 15-17 de diciembre 2009. [Kuala Lumpur]: IEEE, 2009, pp. 815-820. Rec. ITU-T Y.1080. Quality of experience requirements for IPTV services. ITU-T, 2008.

Rec. ITU-T Y.114. One-way transmission time. ITU-T, 2003.

Rec. ITU-T Y.1541. Network performance objectives for IP-based services. ITU-T, 2011. 
RUEDA, D. y RAMOS, Z. Análisis de las alternativas de funcionamiento de la red LTE para la entrega de servicios de IPTV con QoS. Revista Avances, Investigación en Ingeniería. 2013b, vol. 10, núm. 2, pp. 35-48.

RUEDA, D. y RAMOS, Z. Arquitectura funcional para la implementación de Mobile IPTV sobre redes LTE y LTE-Avanced. Revista ITECKNE. 2012, vol. 9, no. 2, pp. 40-51.

RUEDA, D. y RAMOS, Z. Revisión a la implementación del servicio de IPTV sobre redes inalámbricas y móviles con calidad de servicio (QoS). Revista UIS de Ingenierías. 2013a, vol. 12 , no. 1, pp. 39-50.

SHEHADA, M.; FU, B.; THAKOLSRI, S. and KELLERER, W. QoE-based resource reservation for unperceivable video quality fluctuation during Handover in LTE. Consumer Communications and Networking Conference (CCNC), Las Vegas, 11-14 de enero 2013, [Las Vegas]: IEEE, 2013, pp. 171-177. 
